\title{
PPAR- $\gamma$ agonist rosiglitazone protects rat peritoneal mesothelial cells against peritoneal dialysis solution-induced damage
}

\author{
YUN-FANG ZHANG, QI WANG, YAN-YAN SU, JIE-LIN WANG, BAO-JUN HUA, \\ SHEN YANG, JUN-XIA FENG and HONG-YAN LI \\ Center of Kidney Disease, Huadu District People's Hospital, Southern Medical University, \\ Guangzhou, Guangdong 510800, P.R. China
}

Received September 12, 2015; Accepted September 26, 2016

DOI: $10.3892 / \mathrm{mmr} .2017 .6196$

\begin{abstract}
Long-term peritoneal dialysis (PD) leads to ultrafiltration failure (UFF). Peritoneal mesothelial cells, which form the innermost monolayer of the peritoneal cavity, have been shown to regulate various responses, including inflammation, in UFF. The present study was designed to investigate the effect of the peroxisome proliferator-activated receptor- $\gamma$ (PPAR- $\gamma$ ) agonist, rosiglitazone, on peritoneal dialysis solution (PDS)-induced injuries in rat peritoneal mesothelial cells (RPMCs). RPMCs were cultured for different durations and with different concentrations of PDS. The gene expression levels of aquaporin-1 (AQP-1) and zonula occluden-1 (ZO-1) were determined using reverse transcription-quantitative polymerase chain reaction analysis. The protein levels of AQP-1, ZO-1 and PPAR- $\gamma$ were measured using western blot analysis. Interleukin (IL)-6 and IL-8 were detected using ELISA. The RPMCs were damaged by stimulation with $4.25 \%$ PDS for $72 \mathrm{~h}$. The expression levels of AQP-1 and ZO-1 were increased, and the secretion of IL- 6 and IL- 8 were decreased by rosiglitazone. The use of the PPAR- $\gamma$ inhibitor, GW-9662, completely prevented the effects of rosiglitazone. These results indicated that PDS exposure stimulated an inflammatory response in the RPMCs. The PPAR- $\gamma$ activator, rosiglitazone, appeared to relieve the injury by inhibiting inflammation, and regulating the expression of AQP-1 and ZO-1, however further investigations are required to elucidate the potential underlying mechanism.
\end{abstract}

Correspondence to: Dr Hong-Yan Li, Center of Kidney Disease, Huadu District People's Hospital, Southern Medical University, 22 Baohua Road, Huadu, Guangzhou, Guangdong 510800, P.R. China

E-mail: lihy0726@126.com

Key words: peritoneal dialysis solution, aquaporin-1, zonula occluden-1, peroxisome proliferator-activated receptor- $\gamma$ inhibitor, rosiglitazone

\section{Introduction}

Peritoneal dialysis (PD) is an efficient kidney replacement therapy for patients with end-stage renal failure (ESRF). Its lower infection rates and reduced costs significantly improve quality of life and increase the survival rates of patients (1). However, the peritoneal membrane, which is directly and continuously exposed to the peritoneal dialysis solution (PDS) exhibits structural and functional alterations following long-term exposure to PD, and causes a decline in peritoneal ultrafiltration capacity, eventually resulting in ultrafiltration failure (UFF) (2). The potential factors responsible for UFF are complex, predominantly including the peritonitis caused by repeated microbial infection and the chronic aseptic inflammation caused by non-physiological dialysis fluid (3). The physiological morphology of the peritoneum eventually evolves showing loss of the mesothelial cells layer, abnormal proliferation of connective tissue, angiogenesis, obliterating vasculopathy, submesothelial fibrosis and calcification, resulting in dysfunction of the exchange between the blood and dialysate (4). Peritoneal mesothelial cells (PMCs) are the most important cell populations of the peritoneum, and are critical in the maintenance of peritoneal homeostasis, immune surveillance, antigen presentation, inflammation and wound healing, regulating the structure and function of the peritoneum (5).

The expression of aquaporin-1 (AQP-1) and zonula occluden-1 (ZO-1) on the PMCs modulates ultrafiltration during PD through different mechanisms, and are important indicators for the evaluation of peritoneal ultrafiltration function $(6,7)$. As water constitutes the most important component of all viable cells, water uptake and discharge are two of the most basic activities in life. It has been reported that there are two ways for water to cross the cell membrane; one being through diffusion, which is affected by temperature, and the other being via the water-selective AQP-1 channel (6). The water transport mediated by AQP-1 accounts for $~ 90 \%$ of the overall ultrafiltration (8). ZO-1-associated tight junction formation is considered to be important for maintaining the osmotic pressure gradient between the peritoneal capillaries and the dialysate (7). Therefore, abnormal expression of AQP-1 and ZO-1 leads to degenerated function of the peritoneum. 
Peroxisome proliferator-activated receptor- $\gamma$ (PPAR- $\gamma$ ) is a member of a nuclear hormone receptor family, which regulates various metabolic pathways as a transcription factor. It is crucial in the regulation of cellular inflammation, fibrosis and endothelial function (9). Thiazolidinedione, similar to rosiglitazone is a selective PPAR- $\gamma$ activator, which is used extensively in the treatment of type 2 diabetes mellitus (10). Previous reports have demonstrated that pretreatment with rosiglitazone results in decreased inflammation and peritoneal thickness in a lipopolysaccharide-induced peritonitis rat model (11). Aramwit et al (12) found that, in patients receiving continuous ambulatory peritoneal dialysis who were administered with rosiglitazone ( $2 \mathrm{mg}$ BID) for 12 weeks, the total body water and extracellular fluid increased significantly (12).

The present study was designed to investigate the potential protective effects of the PPAR- $\gamma$ agonist, rosiglitazone, on the peritoneal alterations in a PDS-induced RPMC model.

\section{Materials and methods}

Materials. Dulbecco's modified Eagle's medium (DMEM)/nutrient mixture F12 (1:1; DMEM/F12) medium and fetal bovine serum (FBS) were obtained from Invitrogen; Thermo Fisher Scientific, Inc. (Waltham, MA, USA). PDS $(1.5,2.5$ and $4.25 \%)$ were from Baxter Healthcare Co., Ltd. (Shanghai, China). Rosiglitazone was from Molekula $\mathrm{GmbH}$ (Nienburg/Weser, Germany), and 15d-PGJ2 and GW-9662 were purchased from Cayman Chemical Company (Ann Arbor, MI, USA). Monoclonal antibodies against Vimentin (Vim), CD45, GAPDH and AQP-1 were from Abcam (Cambridge, UK). Monoclonal antibody against Factor VIII was from Bioss, Inc. (Woburn, MA, USA). Monoclonal antibodies against ZO-1 and PPAR- $\gamma$, and secondary horseradish peroxidase (HRP)-conjugated antibodies were from Santa Cruz Biotechnology, Inc. (Santa Cruz, CA, USA).

RPMC isolation and culture. The RPMCs were isolated and cultured, as described previously (13). Briefly, male Sprague-Dawley rats (150-250 g) were purchased from the Animal Experimental Center of Sun Yat-Sen University, (Guangzhou, China). The rats $(\mathrm{n}=40)$ were housed in plastic cages on bedding of chips. All rats were given the distilled water to drink and allowed free access to the water and pellet food. All rats were maintained at $25^{\circ} \mathrm{C}$ with 12 -h light/12-h dark cycle. The mice were sacrificed with pentobarbital natrium $(30 \mathrm{mg} / \mathrm{kg}$ at day 7 after surgery. All procedures were followed the rules of the Southern Medical University (Guangzhou, China) Animal Experiment Committee. Individual rats were injected intraperitoneally with $30 \mathrm{ml}$ of $0.25 \%$ trypsinase- $0.02 \%$ EDTA- $\mathrm{Na}_{2}$, and $5 \mathrm{ml}$ abdominal fluid of individual rats was collected $2 \mathrm{~h}$ later, which was centrifuged at $820 \mathrm{x} g$ for $10 \mathrm{~min}$. The cells were washed in PBS and suspended in DMEM/F12 medium with $10 \%$ (v/v) FBS, and were then cultured in $25 \mathrm{~cm}^{2}$ tissue culture flasks at $37^{\circ} \mathrm{C}$ in a humidified $5 \% \mathrm{CO}_{2}$ atmosphere. The cells were passaged every 3-5 days, and RPMCs from the second and third passages at $80 \%$ confluence were used for the following experiments.

Immunocytochemical staining. RPMCs at a density of $2 \times 10^{5} /$ well were cultured overnight on glass cover-slips in
$3.5 \mathrm{~cm}$-diameter tissue culture plates. For immunostaining for DAPK-and RASSF1A, antigen retrieval was performed using microwaves for $10 \mathrm{~min}$. The slides were exposed to Power Block (BioGenex Laboratories, San Ramon, CA, USA) for $45 \mathrm{~min}$. The cells were fixed in cold $4 \%$ paraformaldehyde overnight, permeabilized with $0.5 \%$ Triton X-100 and then blocked with $5 \%$ normal goat serum (BioGenex Laboratories). The cells were then incubated with the following primary antibodies: Anti-Vim (1:150; cat. no. MMS-464S), anti-CD45 (1:150; cat. no. PB9096) and anti-Factor VIII (1:150; cat. no. PRO-318) in $1 \% \mathrm{BSA}$ at $4^{\circ} \mathrm{C}$ overnight. Following washing three times in PBS, the slips were incubated with HRP-conjugated secondary antibodies for $2 \mathrm{~h}$ at $37^{\circ} \mathrm{C}$. The reaction was terminated with 3,3-diaminobenzidine. Images of the results were captured (magnification, x400) using a Nikon ECLIPSE 80i microscope (Nikon, Tokyo, Japan). The optical densities and numbers of DAPK- and RASSF1A-positive cells were counted in five randomly selected fields per sample in spinal anterior horn, and quantification was performed using Image-Pro Plus version 6.0 (Media Cybernetics, Inc., Rockville, MD, USA). Histology and immunohistochemistry for each marker were performed simultaneously in all samples and in negative controls without primary antibodies.

Western blot analysis. Western blot analysis was performed according to standard western blot procedures as previously described (9). The proteins were extracted using Pro-Pprep protein extraction solution (Intron Biotechnology, Inc., Seongnam, South Korea) from the frozen kidney tissues. The protein concentration was measured using Dc Protein Assay kit (Bio-Rad Laboratories, Hercules, CA, USA). Proteins (30 g per lane) were separated by $10 \%$ SDS-PAGE and then transferred onto nitrocellulose membranes (Bio-Rad Laboratories, Inc., Hercules, CA, USA). Following blocking in 5\% nonfat milk, the membranes were incubated with the following primary antibodies: AQP-1 (1:300; cat. no. sc-32739), ZO-1 (1:300; cat. no. 33-9100), PPAR- $\gamma$ (1:300; cat. no. sc-7273) and GAPDH (1:1,000; cat. no. E12-052-1) overnight at $4^{\circ} \mathrm{C}$, followed by incubation with the anti-rabbit HRP-conjugated secondary antibodies (cat. no. 1721011; Bio-Rad Laboratories) for $1 \mathrm{~h}$ at room temperature. Chemiluminescence was detected using ECL Advance Western Blotting Detection reagents (GE Healthcare Life Sciences, Chalfont, UK). The relative expression levels of AQP-1 and ZO-1 were quantified using ImageJ version 1.45 .

Reverse transcription-quantitative polymerase chain reaction (RT-qPCR) analysis. Total RNA was extracted using TRIzol reagent (Ambion; Thermo Fisher Scientific, Inc.) according to manufacturer's protocols. The cDNAs used to examine the expression of AQP-1 and ZO-1 were synthesized using a PrimeScript RT reagent kit (Takara Bio, Inc., Otsu, Japan) according to manufacturer's protocols. The expression of AQP-1 and ZO-1 were examined using SYBR ${ }^{\circledR}$ Premix Ex Taq II (Takara Bio, Inc.), and GAPDH served as an internal reference. PCR was performed using $1 \mu \mathrm{l}$ cDNA in a total volume of $25 \mu \mathrm{l}$ in the presence of $12.5 \mu 12 \mathrm{xiQ}$ Supermix (Bio-Rad Laboratories), 200 nM AQP-1, or ZO-1 primer set. PCR conditions were as follows: 40 cycles at $95^{\circ} \mathrm{C}$ for $30 \mathrm{sec}$, $60^{\circ} \mathrm{C}$ for $1 \mathrm{~min}$ using iCycler iQ qPCR detection system 


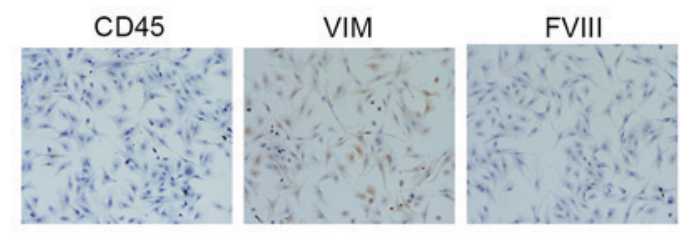

Figure 1. Identification of RPMCs in the cultured cells. RPMCs were detected using immunocytochemical staining with specific markers. CD45, cytokeratin 45, VIM, Vimentin; FVIII, factor VIII.

(Bio-Rad Laboratories). All experiments were performed in duplicate and repeated twice. The results are presented as the fold induction, determined using the $2^{-\Delta \Delta C q}$ method (14). The primers for AQP-1 were: Sense 5'-GCACAATGGGCTCTA TCTTC-3' and antisense 5'-GACAGTCGTTC-TATGGTGGG -3'. The primers for ZO-1 were: Sense 5'-AAAAGTGAACCA CGAG-ATGCT-3' and antisense 5'-AAAGGTAAGGGACTG GAGATGA-3'. The primers for $\beta$-actin were: Sense $5^{\prime}$-GGC AAGTTCAATGGCACAGT-3' and antisense 5'-AAGGTG GAGGAATGGGAGTT-3'.

ELISA. With the collected cell culture supernatant, the concentrations of IL-6 and IL-8 were determined using commercial ELISA kits (R\&D Systems, Inc., Minneapolis, MN, USA) according to the manufacturer's protocol. Three independent assessments were performed.

Statistical analysis. The results of the analyses are expressed as the mean \pm standard deviation. Statistical analaysis was performed using Statview version 5.0 (SAS Intelligence, Cary, NC, USA) Statistically significant differences between groups were identified using one-way analysis of variance, followed by Student's $t$-test (paired). $\mathrm{P}<0.05$ was considered to indicate a statistically significant difference.

\section{Results}

Identification of RPMCs. The identification of the cultured cells as RPMCs was based on their typical markers, CD45, Vim, Desmin and Factor VIII. Immunocytochemical staining of the cultured cells was performed, and the cells were then examined under an optical microscope. Of the characterized markers, it was found that the cells were positively stained for CD45 and Vim, and negatively stained for Desmin and Factor VIII (Fig. 1).

mRNA and protein levels of AQP-1 and ZO-1 in RPMCs treated with $4.25 \%$ PDS for different durations. To observe the effects of PDS on the levels of AQP-1 and ZO-1 in the RPMCs, the RPMCs were cultured with $4.25 \%$ PDS for 0 , 12, 24, 48 and $72 \mathrm{~h}$. Compared with the expression at $0 \mathrm{~h}$, the gene expression of AQP-1 was significantly increased at $24 \mathrm{~h}$, however, a decreasing trend was observed at 48 and $72 \mathrm{~h}$, and this decrease was statistically significantly at $72 \mathrm{~h}$. The mRNA levels of ZO-1 were significantly reduced 24, 48 and $72 \mathrm{~h}$ following treatment with $4.25 \%$ PDS, compared with the level at $0 \mathrm{~h}$ (Fig. 2A). As shown in Fig. 2B, compared with the levels at $0 \mathrm{~h}$, the protein levels of AQP-1 were significantly decreased at $72 \mathrm{~h}$, and the protein expression levels of ZO-1 were significantly decreased at 24,48 and $72 \mathrm{~h}$.
A
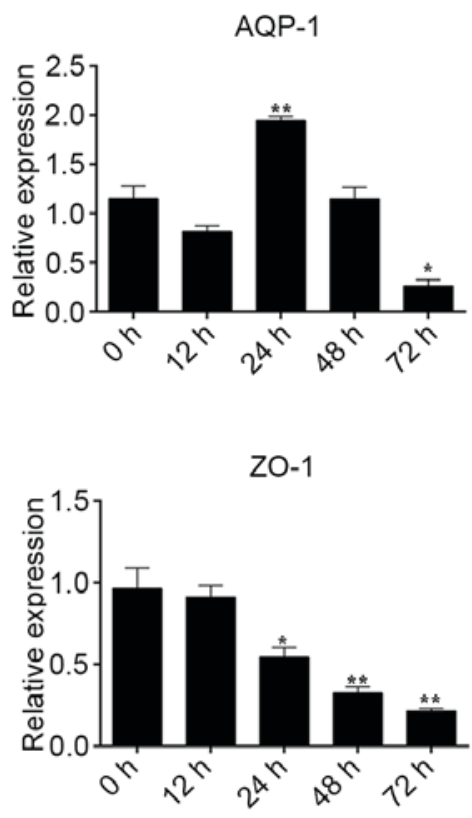

B

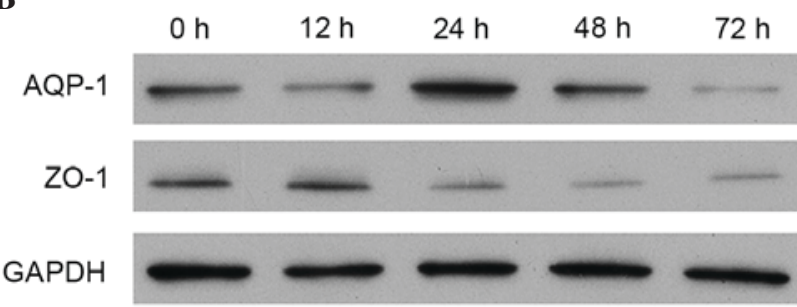

Figure 2. Expression of AQP-1 and ZO-1 in RPMCs treated with 4.25\% PDS for $0,12,24,48$ and 72 h. (A) Gene expression levels of AQP-1 and ZO-1 in the RPMCs were determined using reverse transcription-quantitative polymerase chain reaction analysis. (B) Protein levels of AQP-1 and ZO-1 in the RPMCs were determined using western blot analysis. Values are presented as the mean + standard deviation $(n=3)$. ${ }^{*} \mathrm{P}<0.05$ and ${ }^{* *} \mathrm{P}<0.01$, vs. group treated with $4.25 \%$ PDS for $0 \mathrm{~h}$ (0 h group). AQP-1, aquaporin-1; ZO-1, zonula occluden-1; RPMCs, rat peritoneal mesothelial cells; PDS, peritoneal dialysis solution.

mRNA and protein levels of AQP-1 and ZO-1 in RPMCs treated with different concentrations of PDS for $72 h$. To evaluate the effect of PDS on the levels of AQP-1 and ZO-1 levels in RPMCs, the RPMCs were cultured with different PDS concentrations of $0,1.5,2.5$ and $4.25 \%$ for $72 \mathrm{~h}$. Compared with the controls (PDS $0 \%$ ), the gene expression levels of AQP-1 and ZO-1 showed a decreased trend, and these decreases were statistically significantly in the $4.25 \%$ group (Fig. 3A). Consistent with the results of the RT-qPCR analysis, the protein expression levels of AQP-1 and ZO- 1 were significantly reduced in the $4.25 \%$ group, as demonstrated by the results of the western blot analysis (Fig. 3B). 
A

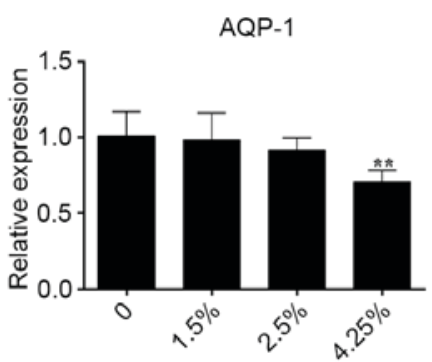

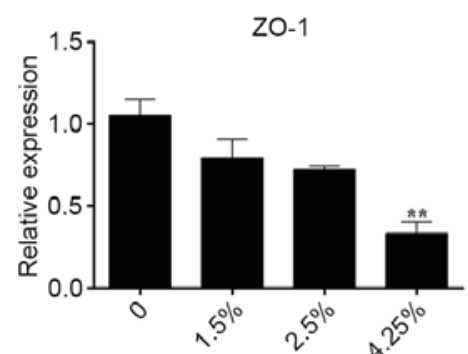

$4.25 \%$

B

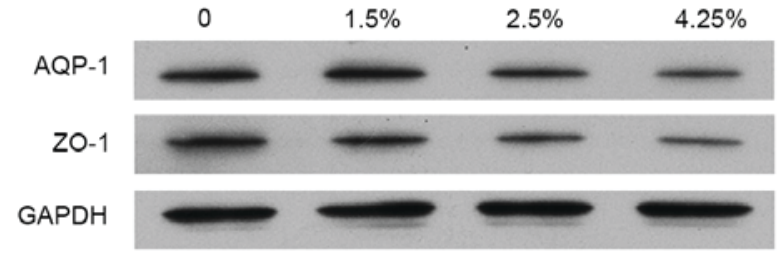

Figure 3. Expression of AQP-1 and ZO-1 in RPMCs treated with different concentrations of PDS for $72 \mathrm{~h}$. (A) mRNA levels of AQP-1 and ZO-1 in RPMCs were determined using reverse transcription-quantitative polymerase chain reaction analysis. (B) Protein expression of AQP-1 and ZO- 1 in the RPMCs were determined using western blot analysis. Values are presented as the mean + standard deviation $(\mathrm{n}=3)$. ${ }^{* *} \mathrm{P}<0.0$, vs. 0\% PDS group. AQP-1, aquaporin-1; ZO-1, zonula occluden-1; RPMCs, rat peritoneal mesothelial cells; PDS, peritoneal dialysis solution.

A

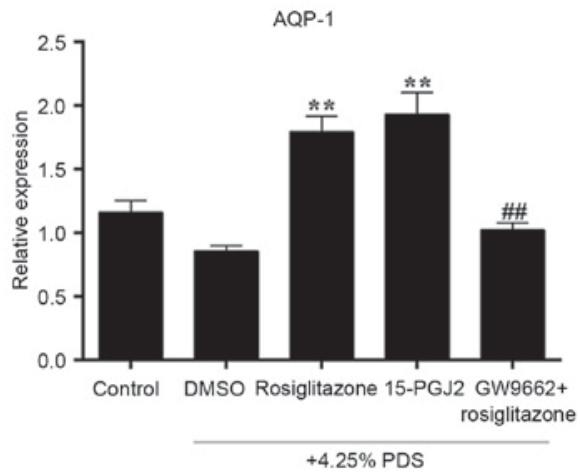

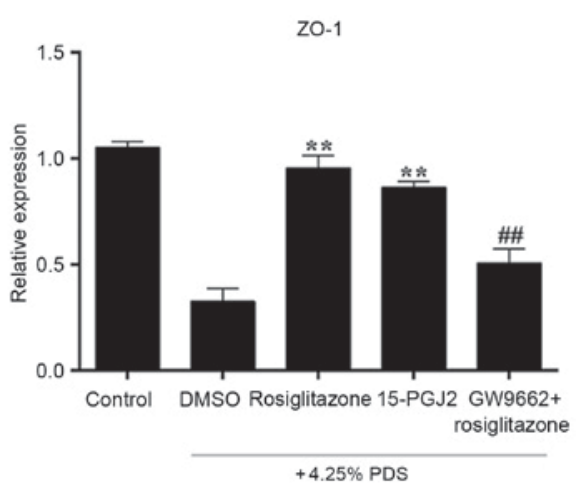

$+4.25 \%$ PDS

B

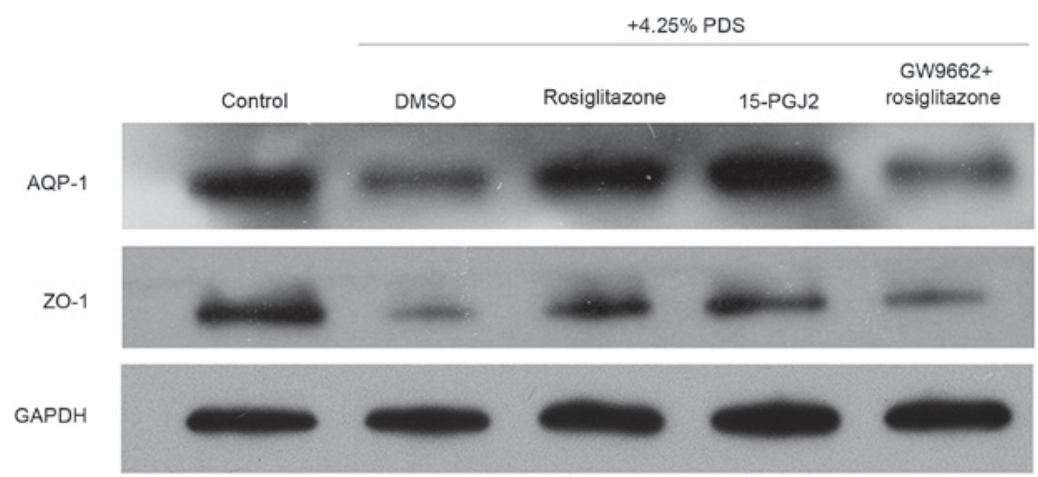

Figure 4. Effects of rosiglitazone on the mRNA and protein expression of 4.25\% PDS-induced AQP-1 and ZO-1. The five groups assessed were: Control group, RPMCs cultured with normal medium only; DMSO group, RPMCs cultured with 0.1\% DMSO and 4.25\% PDS for $72 \mathrm{~h}$; Rosiglitazone group, RPMCs pre-treated with $15 \mu \mathrm{mol} / 1$ rosiglitazone dissolved in $0.1 \%$ DMSO for $2 \mathrm{~h}$, then with $4.25 \%$ PDS for $72 \mathrm{~h}$; 15 -PGJ2 group, RPMCs pre-treated with $5 \mu$ mol/1 15-PGJ2 (PPAR- $\gamma$ activator) dissolved in $0.1 \%$ DMSO for $2 \mathrm{~h}$, then with $4.25 \%$ PDS for $72 \mathrm{~h}$; GW9662 + rosiglitazone group, RPMCs pre-treated with $3 \mu \mathrm{mol} / 1$ GW9662 (PPAR- $\gamma$ inhibitor) for $1 \mathrm{~h}$, then with $15 \mu \mathrm{mol} / 1$ rosiglitazone for $2 \mathrm{~h}$, followied by $4.25 \%$ PDS for $72 \mathrm{~h}$. (A) mRNA levels of AQP-1 and ZO-1 in RPMCs were determined using reverse transcription-quantitative polymerase chain reaction analysis. (B) Protein expression of AQP-1 and ZO-1 in RPMCs, determined using western blot analysis. Values are presented as the mean + standard deviation $(\mathrm{n}=3)$. ${ }^{* *} \mathrm{P}<0.01$, vs. DMSO group; ${ }^{\# \#} \mathrm{P}<0.01$, vs. rosiglitazone group. AQP-1, aquaporin-1; ZO-1, zonula occluden-1; RPMCs, rat peritoneal mesothelial cells; PDS, peritoneal dialysis solution; PPAR- $\gamma$, peroxisome proliferator-activated receptor- $\gamma$.

Effects of rosiglitazone on the $M R N A$ and protein expression of $4.25 \%$ PDS-induced AQP-1 and ZO-1. To evaluate the effect of rosiglitazone in the PDS-induced RPMC model, the
RPMCs were pre-treated with $15 \mu \mathrm{mol} / 1$ rosiglitazone or with $5 \mu \mathrm{mol} / 1$ 15-PGJ2, another PPAR- $\gamma$ agonist, for $2 \mathrm{~h}$, or were pre-treated with $15 \mu \mathrm{mol} / \mathrm{l}$ rosiglitazone for $2 \mathrm{~h}$ following 


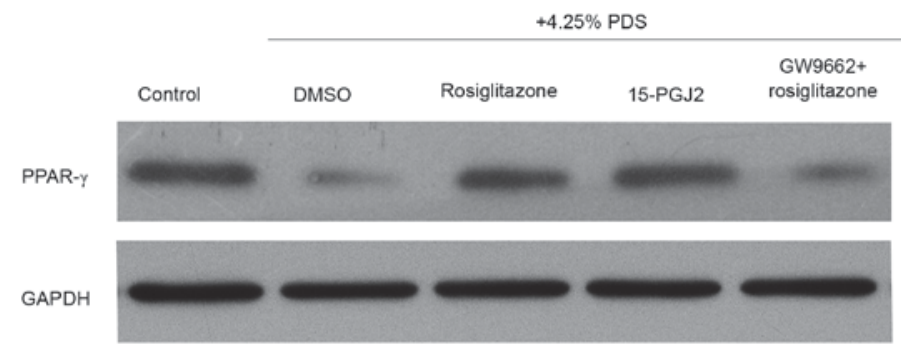

B

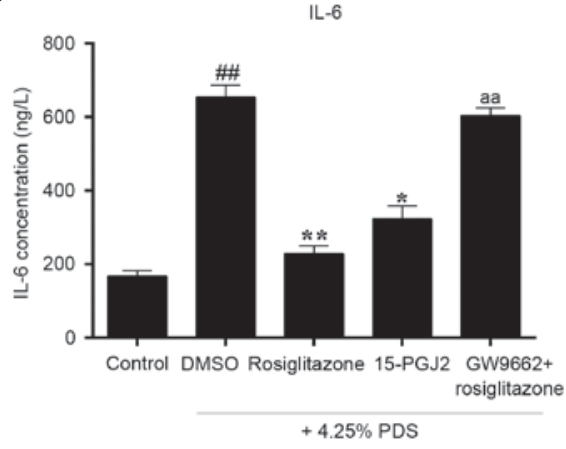

$14-8$

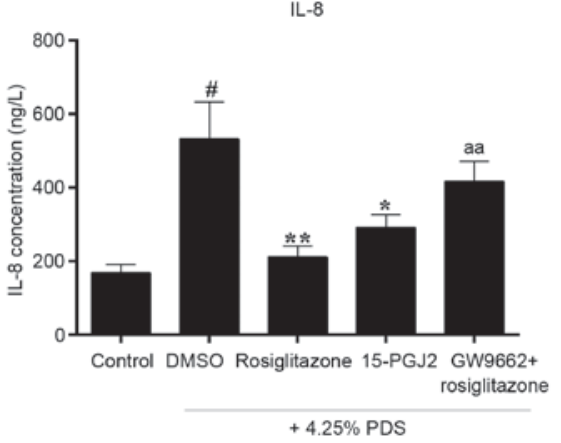

Figure 5. Effects of rosiglitazone on the protein levels of PPAR- $\gamma$ and the secretion of IL- 6 and IL-8. (A) Protein levels of PPAR- $\gamma$ in rat peritoneal mesothelial cells were determined using western blot analysis. (B) Secretion of IL-6 and IL-8 in RPMCs was determined using ELISA. Values are presented the mean + standard deviation $(\mathrm{n}=3) .{ }^{\#} \mathrm{P}<0.05$ and ${ }^{\# \#} \mathrm{P}<0.01$, vs. control group; ${ }^{*} \mathrm{P}<0.05$ and ${ }^{* *} \mathrm{P}<0.01$, vs. DMSO group; ${ }^{\text {aa }} \mathrm{P}<0.01$, vs. rosiglitazone group. $\mathrm{PDS}$, peritoneal dialysis solution; IL, interleukin; PPAR- $\gamma$, peroxisome proliferator-activated receptor- $\gamma$.

pre-treatment with $3 \mu \mathrm{mol} / 1$ GW9662, a PPAR- $\gamma$ inhibitor, for $1 \mathrm{~h}$. As shown in Fig. 4A, the gene expression levels of AQP-1 and ZO-1 in the 4.25\% PDS-induced RPMCs were significantly enhanced by the PPAR $-\gamma$ agoinst, rosiglitazone, compared the with model group (DMSO+4.25\% PDS). The effects of the other PPAR- $\gamma$ activator, 15-PGJ2, were consistent with that of rosiglitazone. By contrast, the mRNA levels of AQP-1 and ZO-1 in the RPMCs pre-treated with rosiglitazone and the PPAR- $\gamma$ inhibitor were reduced significantly, compared with the RPMCs pre-treated with rosiglitazone only. As shown in Fig. 4B, further examination using western blot analysis revealed that the protein levels of AQP-1 and ZO-1 were significantly increased in the rosiglitazone group and 15-PGJ2 group, compared with the model group. The use of GW-9662 appeared to completely prevent the effects of rosiglitazone.

Effects of rosiglitazone on the protein levels of PPAR- $\gamma$ and the secretion of $I L-6$ and $I L-8$. Previous studies have suggested that PPAR $-\gamma$ is a target of rosiglitazone. In the present study, it was found that rosiglitazone was important in the injury induced by PDS in the RPMCs. The present study then investigated whether PPAR- $\gamma$ is involved in the PDS-induced RPMC model. As shown in Fig. 5A, compared with the control group, the protein levels of PPAR- $\gamma$ were reduced by $4.25 \%$ PDS. The expression of PPAR- $\gamma$ was significantly elevated in the rosiglitazone group and the 15-PGJ2 group, compared with the model group (DMSO+4.25\% PDS). The use of GW-9662 did not completely prevent the effects of rosiglitazone. The present study further investigated whether rosiglitazone has effects on the $4.25 \%$ PDS-induced secretion of the IL- 6 and IL- 8 inflammatory cytokines in the RPMCs. As shown in Fig. 5B, the levels of IL-6 and IL- 8 in the cellular supernatant of the model group were significantly increased, compared with those in the control group. However, pre-treatment of the cells with rosiglitazone or $15-\mathrm{PGJ} 2$ reduced these two inflammatory cytokines, compared with the model group. Compared with the rosiglitazone group, the decreases in the levels of IL-6 and IL-8 induced by rosiglitazone were reversed by GW-9662.

\section{Discussion}

PD is an important treatment modality for patients with ESRF, however, a decrease in ultrafiltration function and UFF are common complications in patients receiving long-term PD, and are a leading cause of PD dropout (4). The impaired ultrafiltration leads to the retention of body water and sodium, increases the risk of cardiovascular disease and results in poor prognosis, particularly in patients with loss of residual renal function (15).

AQPs are a group of water-selective channel proteins. They are important in maintaining fluid balance in an organism. Until now, 14 isoforms have been identified. AQP-1 was the first member to be identified in this family (16). AQP-1 has been reported to be responsible for osmotically driven water movement across the peritoneal membrane (16). It is reported that high glucose dialysate upregulates the expression of AQP-1 in uremic rats, however, it is not accompanied by an increase in ultrafiltration. Ni et al (17) investigated the effects of the deletion of AQP-1 on the structure of the mouse peritoneum, and found that, compared with AQP-l(+/+) litter mates, AQP-1(-/-) mice had no sodium sieving. The initial, solute-free ultrafiltration decreased by $\sim 70 \%$ and cumulative 
ultrafiltration decreased by almost $50 \%$. Endothelial cells and tight junctions form the basic structures. The structure comprises a complex of a set of protein molecular elements, including transmembrane proteins, predominantly occludins, claudins, in the junction-associated molecules in the protein family composition $(18,19)$. The membrane components of tight junction molecules interact with each other and their neighbors in tight junctions membrane protein polymerization to form stable tight junctions between cells. $\mathrm{ZO}-1$ is the first component to have been confirmed to be closely connected to the protein and belongs to the membrane-associated guanylic acid kinase protein family (20). ZO-1 is predominantly composed of the PDZ domain, Src homologous 3 domain and guanylate kinase domain. ZO-1 functions to maintain epithelial cell polarity, is indirectly involved in the formation of the cytoskeleton, and is one of the important proteins present in cell tight junctions $(20,21)$. In addition, $\mathrm{ZO}-1$ is a signal transducer in the barrier function and metastasis of cancer cells. The present study focused on the regulation of expression and the relevant functions of AQP-1 and ZO-1 in RPMCs, which are key proteins in the peritoneum during $\mathrm{PD}$ and are important indicators for the evaluation of peritoneal ultrafiltration function. In the present study, RPMCs were cultured with $4.25 \%$ PDS for $0,12,24,48$ and $72 \mathrm{~h}$. It was found that the expression of AQP-1 was increased at $24 \mathrm{~h}$, which was possibly due to the adaptive response of the cells to 4.5\% PDS pre-treatment. However, the expression of AQP-1 showed a significant decrease at $72 \mathrm{~h}$, and the expression of ZO-1 was significantly decreased at 24, 48 and $72 \mathrm{~h}$. A previous study showed that the expression of AQP-1 in human peritoneal mesothelial cells (HPMCs) was enhanced by glucose and other osmotically active agents, and the potential mechanism involved the hypertonic characteristics of PDS stablizing the AQP-1 protein and extending its biological half-life $(22,23)$. However, Fusshoeller (4) found that the expression of AQP-1 in peritoneal mesothelial cells gradually decreased with prolonged dialysis duration (4). The results of the present study confirmed this finding, therefore; $72 \mathrm{~h}$ was selected as the treatment duration for the PDS-induced damage model. Subsequently, the RPMCs were cultured with $0,1.5,2.5$ and $4.25 \%$ of PDS for $72 \mathrm{~h}$. It was found that the expression levels of AQP-1 and ZO-1 were reduced significantly in the $4.25 \%$ group. These results indicated that RPMCs cultured with $4.5 \%$ PDS for $72 \mathrm{~h}$ were damaged by assessment of the expression levels of AQP-1 and ZO-1.

PPARs belong to the nuclear hormone receptor super family, which regulates transcription by binding to retinoid $\mathrm{X}$ receptor, which is in turn bound to DNA in various cell types (9). PPARs have three phenotypes and PPAR- $\gamma$ is one of these. It has been reported that the decreased expression of PPAR- $\gamma$ may be important in activating the pathogenesis of UFF, and the ligands of PPAR- $\gamma$ can inhibit or reverse the development of UFF (24). Rosiglitazone is a synthetic ligand of PPAR- $\gamma$, which can have effects on inflammation, fibrosis and angiogenesis. Sandoval et al (25) revealed that PPAR- $\gamma$ agonist, rosiglitazone, ameliorated peritoneal membrane damage in a mouse model of PD, the underlying mechanism being that rosiglitazone reduced the accumulation of advanced glycation end-products, preserved the mesothelial monolayer, decreased the number of invading mesothelial cells, and reduced fibrosis and angiogenesis. Furthermore, rosiglitazone treatment augments the levels of the anti-inflammatory cytokine, IL-10, and increases the recruitment of $\mathrm{CD} 4^{+} \mathrm{CD} 25^{+} \mathrm{FoxP} 3^{+}$cells. Sauter et al (26) showed that the activation of PPAR $-\gamma$ by glitazones reduces the expression and release of tumor necrosis factor $\alpha$-stimulated monocyte chemoattractant protein-1 in HPMCs (26). Yao et al (27) found that high concentrations of glucose and glucose degradation products in PDS stimulate an inflammatory response in HPMCs, and that rosiglitazone decreases fibrosis by inhibiting the inflammatory factors, IL-6 and IL-8, and regulating the transforming growth factor/small mothers against decapentaplegic signaling pathway (27). In the present study, it was found that rosiglitazone and another PPAR- $\gamma$ agonist, 15-PGJ2, attenuated the damage induced by $4.25 \%$ PDS by increasing the expression levels of AQP-1 and ZO-1, however, these effects were reversed by the PPAR- $\gamma$ inhibitor, GW9662. Furthermore, the present study examined the expression of PPAR- $\gamma$ and inflammatory cytokines in RPMCs cultured with $4.25 \%$ PDS for $72 \mathrm{~h}$, and found a decrease in the levels of PPAR- $\gamma$ and increase in the levels of IL-6 and IL-8. However, when the cells were pre-treated with rosiglitazone and 15-PGJ2, the expression of PPAR- $\gamma$ was increased, and the levels of IL- 6 and IL- 8 were reduced. These effects were also reversed by GW9662.

In conclusion, rosiglitazone was found to directly and specifically potentiate AQP-1-mediated water transport and ZO-1-mediated tight junctions, through elevating the levels of PPAR- $\gamma$ and attenuating inflammation in the RPMCs treated with $4.25 \%$ PDS. This suggests the requirement for further investigation for the complications associated with long-term PDS.

\section{Acknowledgements}

This study was funded by the Guangzhou Medical Key Subject Construction Project (grant no. 2013-2015) and the Program of Huadu District Science and Technology, Guangzhou, China (grant no. 13-HDWS2003).

\section{References}

1. Ditsawanon P and Aramwit P: Preserving the peritoneal membrane in long-term peritoneal dialysis patients. J Clin Pharm Ther Aug 17, 2015 (Epub ahead of print).

2. Yuvaraj A, Koshy PJ, Rohit A, Nagarajan P, Nair S, Revathi L and Abraham G: Diagnostic dilemma of ultrafiltration failure in a continuous ambulatory peritoneal dialysis patient. Perit Dial Int 35: 233-234, 2015.

3. Teitelbaum I: Ultrafiltration failure in peritoneal dialysis: A pathophysiologic approach. Blood Purif 39: 70-73, 2015.

4. Fusshoeller A: Histomorphological and functional changes of the peritoneal membrane during long-term peritoneal dialysis. Pediatr Nephrol 23: 19-25, 2008.

5. Ranzinger J, Rustom A and Schwenger V: Membrane nanotubes between peritoneal mesothelial cells: Functional connectivity and crucial participation during inflammatory reactions. Front Physiol 5: 412, 2014.

6. Morelle J and Devuyst O: Water and solute transport across the peritoneal membrane. Curr Opin Nephrol Hypertens 24: 434-443, 2015.

7. Kaneda K, Miyamoto K, Nomura S and Horiuchi T: Intercellular localization of occludins and ZO-1 as a solute transport barrier of the mesothelial monolayer. J Artif Organs 9: 241-250, 2006.

8. Devuyst $\mathrm{O}$ and Rippe B: Water transport across the peritoneal membrane. Kidney Int 85: 750-758, 2014. 
9. Han JY, Kim YJ, Kim L, Choi SJ, Park IS, Kim JM, Chu YC and Cha DR: PPARgamma agonist and angiotensin II receptor antagonist ameliorate renal tubulointerstitial fibrosis. J Korean Med Sci 25: 35-41, 2010.

10. Ma L, Shao Z, Wang R, Zhao Z, Dong W, Zhang J, Zhang X, Sheng S, Ji Z and Zhang J: Rosiglitazone improves learning and memory ability in rats with type 2 diabetes through the insulin signaling pathway. Am J Med Sci 350: 121-128, 2015.

11. Zhang YF, Zou XL, Wu J, Yu XQ and Yang X: Rosiglitazone, a peroxisome proliferator-activated receptor (PPAR)- $\gamma$ agonist, attenuates inflammation via NF-kB inhibition in lipopolysaccharide-induced peritonitis. Inflammation 38: 2105-2115, 2015.

12. Aramwit P, Bunmee P and Supasyndh O: Effectiveness and tolerability of rosiglitazone on insulin resistance and body composition in nondiabetic Thai patients undergoing continuous ambulatory peritoneal dialysis: A 12-week pilot study. Curr Ther Res Clin Exp 70: 377-389, 2009.

13. Nie J, Dou X, Hao W, Wang X, Peng W, Jia Z, Chen W, Li X, Luo N, Lan HY and Yu XQ: Smad7 gene transfer inhibits peritoneal fibrosis. Kidney Int 72: 1336-1344, 2007.

14. Livak KJ and Schmittgen TD: Analysis of relative gene expression data using real-time quantitative PCR and the 2(-Delta Delta C(T)) Method. Methods 25: 402-408, 2001

15. Guo J, Xiao J, Gao H, Jin Y, Zhao Z, Jiao W, Liu Z and Zhao Z: Cyclooxygenase- 2 and vascular endothelial growth factor expressions are involved in ultrafiltration failure. J Surg Res 188 : 527-536.e2, 2014.

16. Devuyst O and Yool AJ: Aquaporin-1: New developments and perspectives for peritoneal dialysis. Perit Dial Int 30: 135-141, 2010.

17. Ni J, Verbavatz JM, Rippe A, Boisdé I, Moulin P, Rippe B, Verkman AS and Devuyst O: Aquaporin-1 plays an essential role in water permeability and ultrafiltration during peritoneal dialysis. Kidney Int 69: 1518-1525, 2006.

18. Qiu L, Chen C, Ding G, Zhou Y and Zhang M: The effects of electromagnetic pulse on the protein levels of tight junction associated-proteins in the cerebral cortex, hippocampus, heart, lung, and testis of rats. Biomed Environ Sci 24: 438-444, 2011.
19. Hawkins BT and Davis TP: The blood-brain barrier/neurovascular unit in health and disease. Pharmacol Rev 57: 173-185, 2005.

20. Ito T, Yorioka N, Kyuden Y, Asakimori Y, Kiribayashi K, Ogawa $\mathrm{T}$ and Kohno N: Effect of glucose polymer on the intercellular junctions of cultured human peritoneal mesothelial cells. Nephron Clin Pract 93: c97-c105, 2003.

21. Leung JC, Chan LY, Li FF, Tang SC, Chan KW, Chan TM, Lam MF, Wieslander A and Lai KN: Glucose degradation products downregulate $\mathrm{ZO}-1$ expression in human peritoneal mesothelial cells: The role of VEGF. Nephrol Dial Transplant 20: 1336-1349, 2005

22. Leitch V, Agre P and King LS: Altered ubiquitination and stability of aquaporin-1 in hypertonic stress. Proc Natl Acad Sci USA 98: 2894-2898, 2001.

23. Nakayama M, Kawaguchi Y, Yamada K, Hasegawa T, Takazoe K, Katoh N, Hayakawa H, Osaka N, Yamamoto H, Ogawa A, et al: Immunohistochemical detection of advanced glycosylation end-products in the peritoneum and its possible pathophysiological role in CAPD. Kidney Int 51: 182-186, 1997.

24. van Hooland S, Boey O, Van der Niepen P, Van den Branden C and Verbeelen D: Effect of short-term rosiglitazone therapy in peritoneal dialysis patients. Perit Dial Int 29: 108-111, 2009.

25. Sandoval P, Loureiro J, González-Mateo G, Pérez-Lozano ML, Maldonado-Rodríguez A, Sánchez-Tomero JA, Mendoza L, Santamaría B, Ortiz A, Ruíz-Ortega M, et al: PPAR- $\gamma$ agonist rosiglitazone protects peritoneal membrane from dialysis fluid-induced damage. Lab Invest 90: 1517-1532, 2010.

26. Sauter M, Kastenmüller K, Belling F, Wörnle M, Ladurner R, Mussack T and Sitter T: Activation of peroxisome proliferator-activated receptor-gamma by glitazones reduces the expression and release of monocyte chemoattractant protein-1 in human mesothelial cells. Mediators Inflamm 2012: 217696 , 2012.

27. Yao Q, Ayala ER, Qian JQ, Stenvinkel P, Axelsson J and Lindholm B: A combination of a PPAR-gamma agonist and an angiotensin II receptor blocker attenuates proinflammatory signaling and stimulates expression of Smad7 in human peritoneal mesothelial cells. Clin Nephrol 68: 295-301, 2007. 